\title{
The Role of MAPT Haplotype $\mathrm{H} 2$ and Isoform $1 \mathrm{~N} / 4 \mathrm{R}$ in Parkinsonism of Older Adults
}

\author{
Guilherme T. Valenca $^{1,2,3,4,6 \odot}$, Gyan P. Srivastava ${ }^{4,6}$, Jamary Oliveira-Filho ${ }^{3}$, Charles \\ C. White ${ }^{4,5,6}$, Lei $\mathrm{Yu}^{7}$, Julie A. Schneider ${ }^{7}$, Aron S. Buchman ${ }^{7}$, Joshua M. Shulman ${ }^{8,9}$, David \\ A. Bennett ${ }^{7}$, Philip L. De Jager ${ }^{4,5,6 *}$
}

\begin{abstract}
1 Movement Disorders Clinic, Roberto Santos General Hospital, Salvador, BA, Brazil, 2 Health Sciences Center, Federal University of Reconcavo of Bahia, Santo Antonio de Jesus, BA, Brazil, 3 Post-Graduate Program in Health Sciences, Federal University of Bahia, Salvador, BA, Brazil, 4 Program in Translational Neuropsychiatric Genomics, Departments of Neurology \& Psychiatry, Brigham and Women's Hospital, Boston, Massachusetts, United States of America, 5 Harvard Medical School, Boston, Massachusetts, United States of America, 6 Program in Medical and Population Genetics, Broad Institute, Cambridge, Massachusetts, United States of America, 7 Rush Alzheimer's Disease Center, Rush University Medical Center, Chicago, Illinois, United States of America, 8 Departments of Neurology, Molecular and Human Genetics, and Neuroscience, and Program in Developmental Biology, Baylor College of Medicine, Houston, Texas, United States of America, 9 Jan and Dan Duncan Neurological Research Institute, Texas Children's Hospital, Houston, Texas, United States of America
\end{abstract}

ه These authors contributed equally to this work.

* pdejager@ rics.bwh.harvard.edu

Citation: Valenca GT, Srivastava GP, Oliveira-Filho J, White CC, Yu L, Schneider JA, et al. (2016) The Role of MAPT Haplotype $\mathrm{H} 2$ and Isoform $1 \mathrm{~N} / 4 \mathrm{R}$ in Parkinsonism of Older Adults. PLOS ONE 11(7): e0157452. doi:10.1371/journal.pone.0157452

Editor: Riqiang Yan, Cleveland Clnic Foundation, UNITED STATES

Received: February 16, 2016

Accepted: May 31,2016

Published: July 26, 2016

Copyright: $\odot 2016$ Valenca et al. This is an open access article distributed under the terms of the Creative Commons Attribution License, which permits unrestricted use, distribution, and reproduction in any medium, provided the original author and source are credited.

Data Availability Statement: All relevant data are within the paper and its Supporting Information files.

Funding: This work is supported by $\mathrm{NIH}$ grants R01AG036836, R01AG015819, K08AG034290, R01NS078009. JMS is additionally supported by a Career Award for Medical Scientists from the Burroughs Wellcome Fund. This work was also supported by CAPES Foundation (Brazil Ministry of Education) research scholarships proc n0: BEX 7281/ 13-7 awarded to GTV and BEX 18178/12-0 awarded to JOF. The funders had no role in study design, data collection and analysis, decision to publish, or preparation of the manuscript.

\section{Abstract}

\section{Background and Objective}

Recently, we have shown that the Parkinson's disease (PD) susceptibility locus MAPT (microtubule associated protein tau) is associated with parkinsonism in older adults without a clinical diagnosis of PD. In this study, we investigated the relationship between parkinsonian signs and MAPT transcripts by assessing the effect of MAPT haplotypes on alternative splicing and expression levels of the most common isoforms in two prospective clinicopathologic studies of aging.

\section{Materials and Methods}

using regression analysis, controlling for age, sex, study and neuropathology, we evaluated 976 subjects with clinical, genotyping and brain pathology data for haplotype analysis. For transcript analysis, we obtained MAPT gene and isoform-level expression from the dorsolateral prefrontal cortex for 505 of these subjects.

\section{Results}

The MAPT H2 haplotype was associated with lower total MAPT expression $(p=1.2 \times 10-14)$ and global parkinsonism at both study entry $(p=0.001)$ and proximate to death $(p=0.050)$. Specifically, haplotype $\mathrm{H} 2$ was primarily associated with bradykinesia in both assessments $(p<0.001$ and $p=0.008)$. MAPT total expression was associated with age and decreases linearly with advancing age $(p<0.001)$. Analysing MAPT alternative splicing, the expression of $1 \mathrm{~N} / 4 \mathrm{R}$ isoform was inversely associated with global parkinsonism $(p=0.008)$ and 
Competing Interests: The authors have declared that no competing interests exist. bradykinesia $(p=0.008)$. Diminished $1 N / 4 R$ isoform expression was also associated with $\mathrm{H} 2(\mathrm{p}=0.001)$.

\section{Conclusions}

Overall, our results suggest that age and $\mathrm{H} 2$ are associated with higher parkinsonism score and decreased total MAPT RNA expression. Additionally, we found that $\mathrm{H} 2$ and parkinsonism are associated with altered expression levels of specific isoforms. These findings may contribute to the understanding of the association between MAPT locus and parkinsonism in elderly subjects and in some extent to age-related neurodegenerative diseases.

\section{Introduction}

Parkinsonian signs, characterized by bradykinesia, rigidity, tremor and gait disturbance, are common in older individuals [1] and are associated with functional impairment, higher risk of cognitive decline, dementia and mortality [2]. Recently, several reports demonstrated an association between pathology in brainstem nuclei and cerebrovascular lesions with mild parkinsonism [3,4]. In previous work, we have shown that the Parkinson's disease (PD) susceptibility locus $M A P T$ is associated with mild parkinsonism in older adults without a clinical diagnosis of $\mathrm{PD}$, but the biological mechanism underlying this association is unknown [5]. The MAPT gene lies on chromosome 17q21, and its encoded protein Tau is involved in microtubule stability and interaction with the cytoskeleton [6]. Six isoforms of this protein, resulting from alternative splicing of exons 2, 3 and 10, are expressed in the adult human brain [7]. In the region containing $M A P T$, a single nucleotide polymorphism (SNP) tags the two major haplotype clades, termed $\mathrm{H} 1$ and $\mathrm{H} 2$, which are defined by a large inversion containing $M A P T$ as well as several other genes. Many studies in the past decade have shown that this locus affects disease risk. MAPT $\mathrm{H} 1$ and its sub haplotype $\mathrm{H} 1 \mathrm{c}$ are reported to be associated with increased risk for certain age-related neurodegenerative diseases, including progressive supranuclear palsy (PSP) [8-11], corticobasal degeneration (CBD) [10,11], Multiple System Atrophy (MSA) [12] and Parkinson's disease (PD) $[13,14]$. These nosological entities also share a clinical spectrum ranging from parkinsonism to dementia. The $\mathrm{H} 2$ haplotype is related in some studies to late onset Alzheimer's disease and frontotemporal dementia risk $[15,16]$; it is also associated with lower brain MAPT expression levels in Alzheimer's disease (AD) patients [15]. Moreover, MAPT alternative splicing and expression have been shown to be important in neuropathological processes $[6,17]$. Our study refines the role of this locus in age-related parkinsonism and examines the hypothesis that the role of the MAPT haplotypes in age-related parkinsonism in older adults from the Religious Orders Study [18] and Rush Memory Aging Project (MAP) [19] may be mediated, in part, by alternative splicing. Exploring the pathophysiology of mild parkinsonism in older individuals can lead us to a better understanding of motor phenotypes in agerelated neurodegenerative diseases.

\section{Materials and Methods}

\section{Subjects selection from Cohorts}

All of the samples used in this project are from autopsied brains obtained from participants in two longitudinal studies of aging, the Religious Orders Study (ROS) [18], started in 1994, and Rush Memory and Aging Project (MAP) [19], started in 1997. Both studies were approved by 
the Institutional Review Board of Rush University Medical Center. All subjects were older and free of known dementia at the time of enrollment and were followed annually with detailed clinical evaluations with a signed informed consent and an Anatomical Gift Act for brain donation. In total, over 3,000 participants have enrolled and 1,200 autopsied to date. Of these, 976 brains were available for the present study.

\section{Genotyping}

DNA was extracted from whole blood, lymphocytes, or frozen postmortem brain tissue. Genotyping was done in three subsets. Genotyping of the first two sets of samples were generated using Affymetrix GeneChip 6.0 platform in 2009 at the Broad Institute's Center for Genotyping, and the third set of samples were genotyped on the Illumina HumanOmniExpress platform in 2012 at the Children's Hospital of Philadelphia. All three datasets were processed through the same quality control analysis and imputation pipeline that has been published in detail previously $[5,20]$. In this study, we investigated the two major MAPT locus haplotypes by tagging the $\mathrm{H} 1$ haplotype and $\mathrm{H} 2$ haplotype with the major allele and minor allele of rs1052553 (MAF $=0.20$ ), respectively. The subhaplotype H1c was tagged with the minor allele of SNP rs242557 $(\mathrm{MAF}=0.38)[21]$.

\section{MAPT gene total expression and Isoforms-level expression using RNA- seq data}

RNA-seq data was generated using postmortem brain tissue (dorsolateral prefrontal cortex) from ROS/MAP subjects. The RNA was extracted from the tissue after the quality control evaluation based on RNA Integrity Number (RIN score) [22]. The library was sequenced using Illumina Hi-Seq with 101bp reads with 4-plex pooling. All the paired-end reads were mapped using TopHat software [23] using the human genome transcriptomic database from Ensemble (http://www.ensembl.org). The Fragment Per Kilobase Per Million (FPKM) was calculated for each isoform of each gene from the human genome. These FPKM values were regarded as expression quantity for each gene and its isoform for further analysis.

\section{Clinical and pathological outcome measures}

The parkinsonian symptoms measurements were performed annually using a 26-items modified version of the motor section of the Unified Parkinson Disease Rating Scale (mUPDRS). Four previously established parkinsonian sign scores (bradykinesia, rigidity, tremor, and gait impaiment) were derived from these 26 items, and a summary global parkinsonism score was constructed by averaging these 4 scores, as previously described (3). We analyzed the first and last measurements of these scores as separate continuous outcomes in multivariable models, but focused primarily on the last measurement because it was taken closest to the time of autopsy.

Postmortem assessment for Lewy bodies, neurofibrillary tangles, neuritic plaque and macro-microscopic cerebral infarcts were collected as detailed in prior publications [4, 24-26].

\section{Statistical Analysis}

In models linking parkinsonism, and its elemental motor traits, with the MAPT haplotype and $M A P T$ expression, parkinsonian outcomes (global parkinsonism, bradykinesia and gait impairment) were skewed to the right and square root transformations were applied prior to the analysis. Similarly, neuritic plaques and neurofibrillary tangles were square root transformed. We dichotomized the measurements of rigidity and tremor due to a excess of 0 values, 
where non-zero values were represented as one and 0 values were represented as 0 in order to increase the signal to noise ratio. In the analysis of MAPT expression, we used number of Fragments Per Kilobase of transcripts per Million mapped reads (FPKM values) to quantify the expression of each isoform and aggregated these FPKM values in order to quantify total gene expression. We summarize continuous variables with means and standard deviations, and dichotomous variables with percentages, for the analyzed population in Table 1.

We applied linear regression for continuous outcomes (bradykinesia, gait and global parkinsonism), and logistic regression models for tremor and rigidity. All models testing for associations with parkinsonism were adjusted for pertinent covariates, including age, sex, study (ROS or MAP) and various brain pathologies, if available at time of measure, including Lewy body (presence or absence), microinfarct (presence or absence), macroinfarct (presence or absence), neuritic plaque and neurofibrillary tangles. In analysis testing association between haplotype and expression, age, sex, cohort and neuropathology were included as covariates in the models. Analyses were done using the base stats package in R version 3.1 (www.r-project.org).

\section{Results}

For the analysis of haplotypes in relation to parkinsonism, a total of 976 subjects with nonmissing genotype and phenotype data were evaluated. A subset of 505 of these subjects also had MAPT RNA sequencing data available (51.7\%) (Table 1). Allele and genotype frequencies for MAPT rs1052553 and rs242557 are shown in S1 Table. The rs1052553 SNP tags the MAPT

Table 1. Cohort characteristics for 976 subjects used for the analysis.

\begin{tabular}{l|r}
\hline Study Subject Characteristics & mean +/- SD or n(\%) \\
\hline Demographics and Clinical Variables & \\
\hline Number of subjects & 976 \\
\hline Age at death & $88.36 \pm(6.5)$ \\
\hline Age at baseline visit & $16.02 \pm(6.91)$ \\
\hline Education & $35.9 \%(351)$ \\
\hline Male & $6.2 \%(60)$ \\
\hline PD diagnosis at death & $51.7 \%(505)$ \\
\hline Subjects with RNA data & $53.2 \%(519)$ \\
\hline Percent in ROS (versus MAP) & \\
\hline mUPDRS scores & $3.31 \pm(1.94)$ \\
\hline Bradykinesia score baseline visit & $3.79 \pm(2.26)$ \\
\hline Bradykinesia score last visit & $3.07 \pm(1.26)$ \\
\hline Global parkinsonism score baseline visit & $3.95 \pm(1.41)$ \\
\hline Global parkinsonism score last visit & $4.02 \pm(2.01)$ \\
\hline Gait score baseline visit & $5.78 \pm(2.05)$ \\
\hline Gait score last visit & $33.3 \%(324)$ \\
\hline Rigidity > 0 baseline visit & $45.8 \%(445)$ \\
\hline Tremor score > 0 baseline visit & $53.4 \%(517)$ \\
\hline Rigidity > 0 last visit & $48.4 \%(471)$ \\
\hline Tremor score > 0 last visit & \\
\hline Post Mortem Pathology & $21.5 \%(210)$ \\
\hline Lewy bodies present in nigra & $0.67 \pm(0.42)$ \\
\hline Neurofibrillary tangles & $0.74 \pm(0.54)$ \\
\hline Neuritic plaques & $35.7 \%(348)$ \\
\hline Macroscopic infarcts & $28.5 \%(278)$ \\
\hline Microscopic infarcts & \\
\hline & \\
\hline
\end{tabular}

doi:10.1371/journal.pone.0157452.t001 
$\mathrm{H} 1 / \mathrm{H} 2$ haplotypes, and the rs242557 SNP tags the MAPT H1c subhaplotype. As expected, advancing age is strongly associated with worsening motor traits (global parkinsonism: $\beta=$ $0.040, \mathrm{p}=2.8 \times 10-8$; S2 Table).

\section{Association of MAPT haplotypes with parkinsonism and its four comprising components}

Using an additive model, we examined the association between the two major MAPT haplotypes and global parkinsonism as well as its four component motor domains: bradykinesia, gait impairment, tremor and rigidity, which are measured at both at baseline and annually thereafter (Table 2)(Fig 1). In our data, we observed an association of the MAPT H2 haplotype with greater global parkinsonism and its component measures of bradykinesia and gait score, but not rigidity or tremor, at the baseline measurement of each subject. By contrast, the MAPT $\mathrm{H} 1 \mathrm{c}$ haplotype is not associated with global parkinsonism or its component measures. Interestingly, the association between the $\mathrm{H} 2$ haplotype and global parkinsonim or gait is no longer detected when considering data obtained at the last evaluation proximate to death nd adjustment for measured neuropathologies (Table 2)(Fig 1), which may be many years after the baseline evaluation. However, the association with bradykinesia persists, with an effect size that is essentially unchanged (Table 2)(Fig 1).

These associations remained following exclusion of subjects with a PD diagnosis: global parkinsonism at baseline adjusting for age, sex, study $(\mathrm{p}<0.001)$, and $\mathrm{p}=0.064$ proximate to death adjusting for age at death, sex, study and pathology. Bradykinesia associations were also not significantly affected: $\mathrm{p}<0.001$ at baseline adjusting for age, sex, study, and $\mathrm{p}=0.019$ proximate to death adjusting for age at death, sex, study and pathology.

In older age, other neuropathologies can also influence parkinsonian signs in this cohort, including macroscopic infarcts $(\beta=0.415, \mathrm{p}<0.0001)$ and Lewy bodies $(\beta=0.27, \mathrm{p}=0.009)$

Table 2. MAPT H1 and $\mathrm{H} 2$ major haplotypes* and MAPT subhaplotype $\mathrm{H} 1 \mathrm{c} *$ * association with global parkinsonism and motor components at baseline $^{\mathrm{a}}$ and last ${ }^{\mathrm{b}}$ measurements.

\begin{tabular}{|c|c|c|c|c|}
\hline \multirow[t]{2}{*}{ Parkinsonism Component } & \multicolumn{2}{|c|}{ rs $1052553 *$} & \multicolumn{2}{|c|}{ rs242557** } \\
\hline & Estimate & Pvalue & Estimate & Pvalue \\
\hline \multicolumn{5}{|l|}{ Baseline measurement } \\
\hline Global Parkinsonism & 0.21 & 0.001 & 0.093 & 0.13 \\
\hline Bradykinesia & 0.37 & $<0.001$ & 0.13 & 0.19 \\
\hline Gait impairment & 0.23 & 0.021 & 0.15 & 0.11 \\
\hline Rigidity & 0.12 & 0.31 & 0.15 & 0.17 \\
\hline \begin{tabular}{l|l} 
& Tremor score \\
\end{tabular} & 0.17 & 0.15 & 0.059 & 0.58 \\
\hline \multicolumn{5}{|l|}{ Last measurement } \\
\hline \begin{tabular}{l|l} 
& Global Parkinsonism \\
\end{tabular} & 0.15 & 0.050 & -0.015 & 0.82 \\
\hline Bradykinesia & 0.32 & 0.008 & 0.086 & 0.45 \\
\hline Gait & 0.18 & 0.12 & -0.012 & 0.91 \\
\hline Rigidity & 0.15 & 0.20 & -0.13 & 0.22 \\
\hline Tremor & -0.10 & 0.38 & -0.068 & 0.52 \\
\hline
\end{tabular}

aSummary of association test using linear regression for global parkinsonism, bradykinesia and gait and logistic regression for dichotomized rigidity and tremor measurement, after adjustment for age,sex, and study.

bSummary of association test using linear regression for global parkinsonism, bradykinesia and gait and logistic regression for dichotomized rigidity and tremor measurement, after adjustment for age, sex, study, Lewy body, neuritic plaque, neurofibrillary tangle, macroscopic infarct and microscopic infarct. 


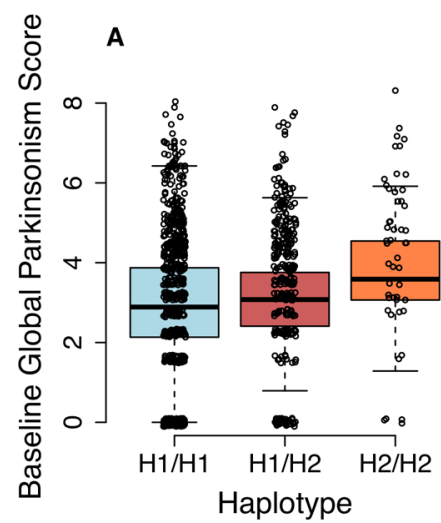

B

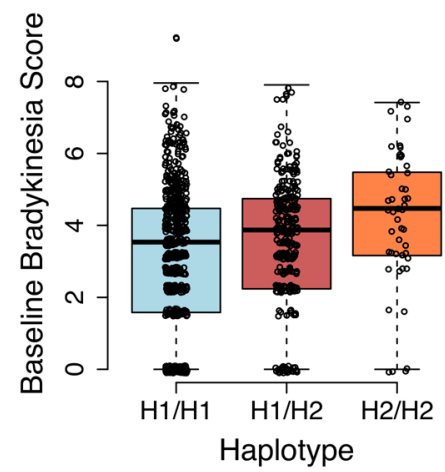

E

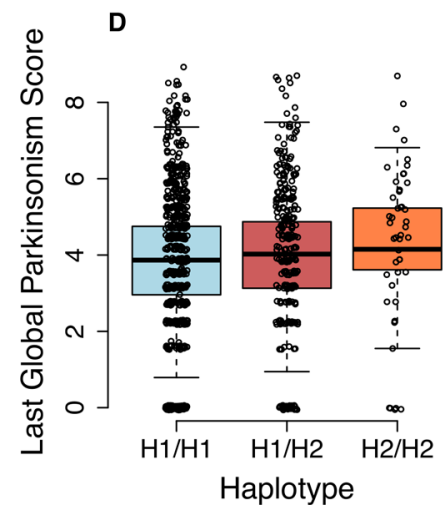

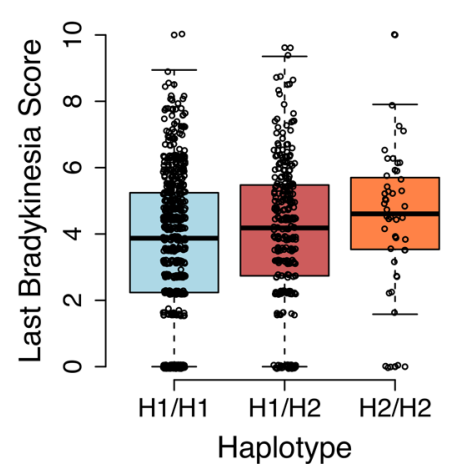

C

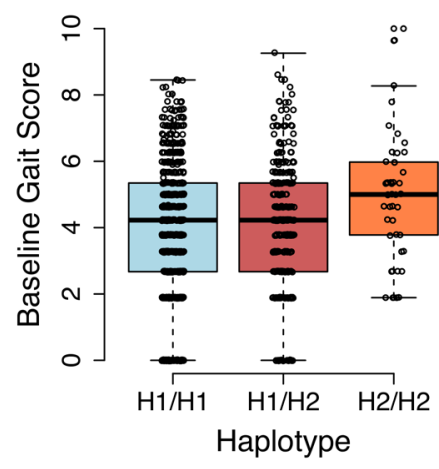

$\mathbf{F}$

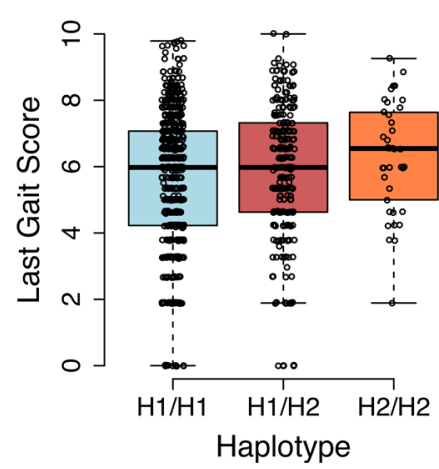

Fig 1. Relation of clinical signs to the H1-H2 haplotype. The tag SNP rs 1052553 is used to differentiate the $\mathrm{H} 1 / \mathrm{H} 2$ haplotypes. The top series of panels $(A, B, C)$ report the association of MAPT genotype with motor traits at the baseline assessment (global parkinsonism, $p=0.001$; bradykinesia, $p<0.001$; gait, $p=0.021$, adjusted for age, sex, study). The bottom series of panels (C, D, E) report the association of MAPT genotype with motor traits at the time of the last available assessment (global parkinsonism, $p=0.050$; bradykinesia, $p=0.008$; gait, $p=0.12$, adjusted for age, sex, study, + path). Each dot represents one subject.

doi:10.1371/journal.pone.0157452.g001

(S2 Table). Once these confounding effects are accounted for in the model, an association with global parkinsonism is seen at the last evaluation and appears to be driven primarily by an effect on bradykinesia $(\mathrm{p}=0.008)$ (Table 2). These intriguing results suggest that the effect of the $\mathrm{H} 2$ haplotype on bradykinesia may be more pronounced earlier in life and that this effect is obscured in later life by the accumulation of neurological insults to the brain such as cerebral infarcts and Lewy bodies.

\section{Association of age, parkinsonian signs and the $\mathrm{H} 1$ and $\mathrm{H} 2$ haplotypes with total MAPT gene brain expression}

In order to gain insight into the association between the MAPT haplotypes and parkinsonian signs, we further analyzed the relationships between the haplotypes, MAPT RNA expression, and parkinsonian phenotypes. First, we found that MAPT total mRNA expression is strongly influenced by age ( $\mathrm{p}<0.001$ age, sex, study and $\mathrm{H} 2$ only; $\mathrm{p}<0.0001$ adjusting for age, sex, study, $\mathrm{H} 2$ and pathology), decreasing linearly with advancing age in all MAPT haplotype categories (S1A Fig). In the analysis testing for evidence of association between haplotypes and total MAPT expression, age, sex, study and neuropathology were included as covariates in the models. Our results show that rs1052553, the tag SNP differentiating the $\mathrm{H} 1 / \mathrm{H} 2$ haplotypes, strongly influences MAPT total expression: as shown in Fig 2A, the $\mathrm{H} 2$ haplotype is strongly 
A

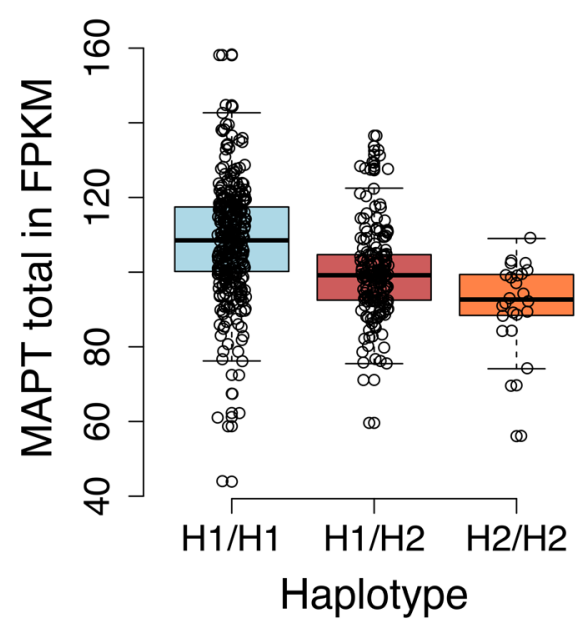

B

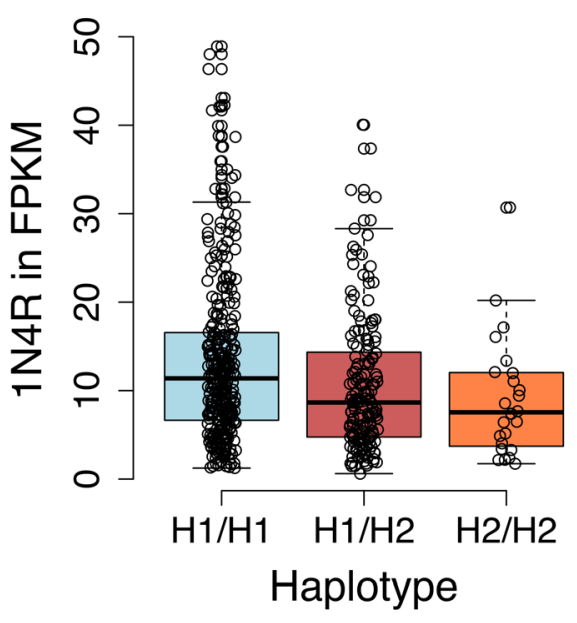

C.
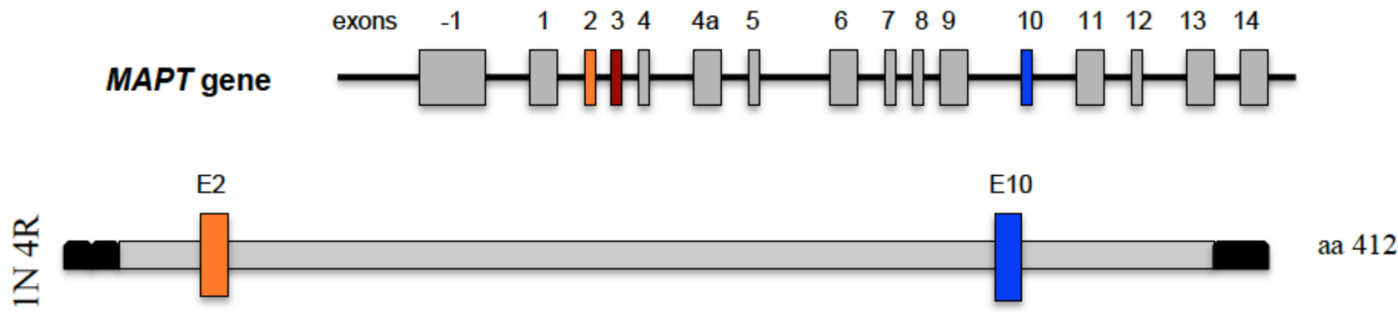

Fig 2. Relation of MAPT expression to the $\mathrm{H} 1 / \mathrm{H} 2$ haplotypes. A dose-dependent effect of the $\mathrm{H} 2$ haplotype is noted. Each dot represents one subject. (A) Total MAPT expression ( $p=1.2 \times 10-14$ adjusted for age, sex, and path); (B) Expression of MAPT isoform $1 \mathrm{~N} 4 \mathrm{R}$, the only isoform to be significantly associated with parkinsonism. $(\mathrm{p}<0.001$ adjusted for age, sex, and study and $\mathrm{p}=0.001$ adjusted for age, sex, study and path); (C) MAPT exon structure and composition of the 1N4R isoform: aa, amino acids; E2, exon 2; E3, exon 3; E10, exon 10.

doi:10.1371/journal.pone.0157452.g002

associated with lower MAPT total expression relative to the H1 haplotype $\left(\mathrm{p}=1.2 \times 10^{-14}\right.$ adjusted for age, sex, and pathology). Although we observed increased global parkinsonism, bradykinesia and gait disturbance scores with decreasing MAPT gene total expression, the association between them was not significant (S2A Fig).

\section{MAPT $\mathrm{H} 2$ haplotype, motor traits and MAPT isoform level analysis}

Since the literature suggests that altered levels or ratios of MAPT RNA isoforms play a role in the susceptibility of age-related neurodegenerative diseases associated with motor impairment [27], we also analysed the relationship between isoform levels to parkinsonism and MAPT haplotypes. Six different isoforms of MAPT are available in our RNA sequencing data. Adjusting for age, sex and brain pathology, we found that the level of the 1N/4R MAPT isoform was inversely associated with global parkinsonism $(\beta=-0.0173 ; \mathrm{p}=0.008$, adjusted for age, sex, study and pathology), bradykinesia $(\beta=-0.0281 ; p=0.008$, adjusted for age, sex, study and pathology) and gait impairment ( $\beta=-0.019 ; \mathrm{p}=0.039)$ at the last visit prior to death. (S2B Fig). After further adjusting for $M A P T$ total level of expression, this isoform remained associated 
with global parkinsonism ( $\beta=-0.0165, p=0.012$, adjusted for age, sex, study and pathology) and bradykinesia ( $\beta=-0.0276, p=0.011$, adjusted for age, sex, study and pathology) (S3 Fig). None of the other MAPT common isoforms expressed in the brain showed association with parkinsonian signs in a model controlling for total expression level and brain pathologies.

We next investigated the relation of the MAPT haplotypes to the level of this RNA isoform. Interestingly, the $\mathrm{H} 2$ haplotype was associated with lower expression of the $1 \mathrm{~N} / 4 \mathrm{R}$ isoform $(\mathrm{p}<0.001$ adjusting for age, sex and study; $\mathrm{p}=0.001$ after adjustment for pathology) (Fig 2B). In addition, age was not associated with the MAPT $1 \mathrm{~N} / 4 \mathrm{R}(\mathrm{p}=0.58)$ (S1B Fig).

We also assessed whether the MAPT RNA 1N/4R isoform expression mediated the effect of the $\mathrm{H} 2$ haplotype on the clinical trait that is most strongly associated with it, bradykinesia. In this analysis, a reduction of effect size by $>10 \%$ is taken as evidence of mediation. Here, we see that inclusion of the $1 \mathrm{~N} / 4 \mathrm{R}$ isoform in the analytic model diminishes the $\beta$ for the $\mathrm{H} 2$ haplotype in relation to bradykinesia by $16 \%$, suggesting that it may mediate, in part, the effect of the $\mathrm{H} 2$ haplotype on bradykinesia. We note that the effect of the $1 \mathrm{~N} / 4 \mathrm{R}$ isoform is reduced when total MAPT gene expression is added to the model ( $\beta$ decreases $3 \%$ with $1 \mathrm{~N} / 4 \mathrm{R}$ ). Thus, while the effects on MAPT expression and splicing may mediate part of the $\mathrm{H} 2$ effect, most of the effects of $\mathrm{H} 2$ and $1 \mathrm{~N} / 4 \mathrm{R}$ on bradykinesia are independent, suggesting that they represent largely independent processes that influence aging-related functional decline ( $\mathrm{p}$ value of $1 \mathrm{~N} /$ $4 \mathrm{R}=0.017$, $\mathrm{p}$ value of $\mathrm{H} 2=0.012$ ).

\section{Discussion}

Parkinsonism, characterized by bradykinesia, rigidity, tremor, gait and balance problems, can commonly be identified during clinical examination of older individuals without prior diagnosis of neurological conditions ${ }^{1}$. These motor features are associated with mild cognitive impairment, increased risk of dementia, PD, depressive symptoms, and cerebrovascular lesions [28]. They are also a significant predictor of mortality [4,29]. Recently, we have identified two genetic variants implicated in PD susceptibility as risk factors for age-related motor impairment; one of these resides in the MAPT locus [5].

Multiple studies have reported the role of haplotype $\mathrm{H} 1$ and subhaplotype $\mathrm{H} 1 \mathrm{c}$ as risk factors for age-related neurodegenerative diseases that share parkinsonian phenotypes, such as PD, MSA, PSP and CBD [8-14]. By contrast, in our cohorts, we observed a significant positive correlation between MAPT H2 haplotype and global parkinsonism. In fact, it is the bradykinetic component of parkinsonism, which is commonly seen in older individuals [2], that drives the association of parkinsonism with MAPT $\mathrm{H} 2$. We also found that the association between parkinsonism and $\mathrm{H} 2$ is stronger in our baseline measurements than in the measurement taken closest to death. This implies that $\mathrm{H} 2$ drives parkinsonism at a younger age and that its effect may wane over time. This observation also may be explained in part by the effect of other factors affecting the same trait, such as other neuropathologies (e.g., the presence of Lewy bodies and macroscopic infarcts), age and possibly epigenetic mechanisms [30,31]. Given that the mean age at death of our subjects is 88 , the effect of $\mathrm{H} 2$ on parkinsonism might be significantly attenuated by the increasing prevalence of these other pathologies. In contrast with bradykinesia and gait, rigidity and tremor were not associated with $\mathrm{H} 2$ haplotype probably because these signs are not frequently observed in older people with parkinsonism [2]. These findings are consistent with our prior analysis in a subset of these subjects [5]. The subhaplotype H1c, tagged by the snp rs242557, which is implicated in tauopathy risk [11], was not associated with any of the parkinsonian measures. Unlike others studies that established the relationship between $\mathrm{H} 1$ and $\mathrm{H} 1 \mathrm{c}$ and age-related neurodegenerative diseases, our results are based in two cohorts of older subjects free of these conditions. Therefore, the association between $\mathrm{H} 2$ 
haplotype and parkinsonism found in our study suggests different mechanisms to explain this phenotype in older individuals.

When we investigated the expression of total MAPT in 505 individuals, we observed that $\mathrm{H} 2$ was strongly associated with lower total MAPT expression compared when compared to H1. This result confirms a recent study [15] where the authors evaluated the association of MAPT haplotypes with brain MAPT gene expression levels in 202 late onset Alzheimer's disease individuals. It was found that the $\mathrm{H} 2$ haplotype was associated with both lower MAPT RNA brain expression at the gene level and late onset AD risk [15]. We also observed that total $M A P T$ brain expression decreases with age in each haplotype category. In fact, the decrease of total MAPT gene expression with advancing age has been described previously [32]. When placed in a single model, both age and the MAPT $\mathrm{H} 2$ haplotype are independently associated with lower total MAPT expression.

Another aspect of MAPT expression addressed in our study was the association of major haplotypes and parkinsonian measures with the six common MAPT isoforms. We observed that the levels of expression of only one of the MAPT isoforms expressed in the human brain were significantly associated with global parkinsonism and bradykinesia scores. Whilst $1 \mathrm{~N} / 4 \mathrm{R}$ isoform was inversely associated with motor findings, we found that $\mathrm{H} 2$ haplotype was associated with increased scores of global parkinsonism and bradykinesia, and lower expression of the transcript $1 \mathrm{~N} / 4 \mathrm{R}$; therefore, the association of $\mathrm{H} 2$ on isoform expression results in competing effects on motor components. In addition, the mediation model suggests that the isoform and $\mathrm{H} 2$ haplotype influence these motor traits largely independently of one another. Interestingly, published data report that $\mathrm{H} 2$ haplotype subjects have 2-folder greater expression of isoforms with segments encoded by exons 2 and 3 [17,33]. Furthermore, Tau isoforms are released at differing rates depending on the contribution of the $\mathrm{N}$ terminus and microtubule binding repeat length $[34,35]$. This imbalanced expression of alternative transcripts is critical for neuronal function and may play a role in motor impairment in older people. Again, the advanced age of the subjects in our cohort could be a decisive factor for this imbalance and hence for this association $[36,37]$.

In summary, our results begin to dissect the complex interplay of different risk factors related to the MAPT locus in its influence of aging-related parkinsonism, mainly through an effect that is manifested clinically as bradykinesia. In addition, we describe the relation of $M A P T$ isoform expression in the frontal cortex in relation to the $\mathrm{H} 1$ and $\mathrm{H} 2$ haplotypes, providing additional data for understanding the role of this important locus in other neurologic diseases.

\section{Supporting Information}

S1 Fig. (a) Relation between age at time of death and MAPT expression stratified by $\mathrm{H1}$ / H2 haplotype ( $\mathbf{p}<\mathbf{0 . 0 0 1}$ age, sex, study and $\mathbf{H} 2$ only; $\mathrm{p}<0.0001$ adjusting for age, sex, study, $\mathrm{H} 2+$ path). (b) Relation between age at time of death and MAPT 1N4R expression stratified by $\mathrm{H} 1 / \mathrm{H} 2$ ( $p=0.58$ adjusting for pathologies). (PDF)

S2 Fig. (a) Relationship between motor traits scores at time prior to death and MAPT total expression (global parkinsonism score, $\mathrm{p}=0.14$; bradykinesia score, $\mathrm{p}=0.45$; gait score, $\mathrm{p}=0.12$ ). (b) Relationship between motor traits scores at time prior to death and MAPT 1N4R total expression (global parkinsonism score, $\mathrm{p}=0.008$; bradykinesia score, $\mathrm{p}=0.008$; gait score, $\mathrm{p}=0.039$ ).

$(\mathrm{PDF})$ 
S3 Fig. Relation between global parkinsonism score at time prior to death and MAPT isoform 1N/4R expression adjusted for MAPT total expression and brain pathology $(\mathbf{p}=\mathbf{0 . 0 1 2})$.

(PDF)

S1 Table. Allele and genotype frequencies for MAPT rs1052553 and rs242557 based on 976 subjects. For rs 1052553 allele A and G correspond to H1 and H2 haplotypes, respectively. For rs242557 allele A corresponds to the H1c haplotype.

(DOCX)

S2 Table. Association of neuropathologies with global parkinsonism score at time of death. Neuropathologies, macroscopic infarcts and Lewy bodies, and age were also associated with global parkinsonism at time prior to death.Based on linear or logistic regression models. (DOCX)

\section{Acknowledgments}

The authors thank the participants in the Rush Memory and Aging Project and Religious Order Study. This work is supported by NIH grants R01AG036836, R01AG015819, K08AG034290, R01NS078009. JMS is additionally supported by a Career Award for Medical Scientists from the Burroughs Wellcome Fund. This work was also supported by CAPES Foundation (Brazil Ministry of Education) research scholarships proc $n^{0}$ : BEX 7281/13-7 awarded to GTV and BEX 18178/12-0 awarded to JOF.

\section{Author Contributions}

Conceived and designed the experiments: GTV GPS PLJ. Analyzed the data: GTV GPS JOF LY CCW. Wrote the paper: GTV GPS JMS PLJ ASB. Revising the version critically for important intellectual content: JAS ASB DAB JMS JOF LY CCW. Final approval of the version to be published: JAS ASB DAB JMS PLJ LY.

\section{References}

1. Louis ED, Bennett DA. Mild Parkinsonian signs: An overview of an emerging concept. Mov Disord. 2007; 22(12):1681-8. PMID: 17534951

2. Hindle JV. Ageing, neurodegeneration and Parkinson's disease. Age Ageing. 2010; 39(2):156-61. doi: 10.1093/ageing/afp223 PMID: 20051606

3. Buchman AS, Shulman JM, Nag S, Leurgans SE, Arnold SE, Morris MC, et al. Nigral pathology and parkinsonian signs in elders without Parkinson disease. Ann Neurol. 2012; 71(2):258-66. doi: 10.1002/ ana.22588 PMID: 22367997

4. Buchman AS, Leurgans SE, Nag S, Bennett DA, Schneider JA. Cerebrovascular disease pathology and parkinsonian signs in old age. Stroke. 2011; 42(11):3183-9. doi: 10.1161/STROKEAHA.111. 623462 PMID: 21885844

5. Shulman JM, Yu L, Buchman AS, Evans DA, Schneider JA, Bennett DA, et al. Association of Parkinson disease risk loci with mild parkinsonian signs in older persons. JAMA Neurol. 2014; 71(4):429-35. doi: 10.1001/jamaneurol.2013.6222 PMID: 24514572

6. Majounie E, Cross W, Newsway V, Dillman A, Vandrovcova J, Morris CM, et al. Variation in tau isoform expression in different brain regions and disease states. Neurobiol Aging. 2013; 34(7):1922 e7-e12. doi: 10.1016/j.neurobiolaging.2013.01.017 PMID: 23428180

7. Niblock M, Gallo JM. Tau alternative splicing in familial and sporadic tauopathies. Biochem Soc Trans. 2012; 40(4):677-80. doi: 10.1042/BST20120091 PMID: 22817715

8. Hoglinger GU, Melhem NM, Dickson DW, Sleiman PM, Wang LS, Klei L, et al. Identification of common variants influencing risk of the tauopathy progressive supranuclear palsy. Nat Genet. 2011; 43(7):699705. doi: 10.1038/ng.859 PMID: 21685912 
9. Rademakers R, Melquist S, Cruts M, Theuns J, Del-Favero J, Poorkaj P, et al. High-density SNP haplotyping suggests altered regulation of tau gene expression in progressive supranuclear palsy. Hum Mol Genet. 2005; 14(21):3281-92. PMID: 16195395

10. Houlden H, Baker M, Morris HR, MacDonald N, Pickering-Brown S, Adamson J, et al. Corticobasal degeneration and progressive supranuclear palsy share a common tau haplotype. Neurology. 2001; 56 (12):1702-6. PMID: 11425937

11. Pittman AM, Myers AJ, Abou-Sleiman P, Fung HC, Kaleem M, Marlowe L, et al. Linkage disequilibrium fine mapping and haplotype association analysis of the tau gene in progressive supranuclear palsy and corticobasal degeneration. J Med Genet. 2005; 42(11):837-46. Epub 2005/03/29. doi: 10.1136/jmg 2005.031377 PMID: 15792962

12. Vilarino-Guell $C$, Soto-Ortolaza AI, Rajput $A$, Mash DC, Papapetropoulos S, Pahwa R, et al. MAPT H1 haplotype is a risk factor for essential tremor and multiple system atrophy. Neurology. 2011; 76(7):670 2. doi: 10.1212/WNL.0b013e31820c30c1 PMID: 21321341

13. Zabetian CP, Hutter CM, Factor SA, Nutt JG, Higgins DS, Griffith A, et al. Association analysis of MAPT H1 haplotype and subhaplotypes in Parkinson's disease. Ann Neurol. 2007; 62(2):137-44. PMID: 17514749

14. Skipper L, Wilkes $K$, Toft M, Baker M, Lincoln S, Hulihan M, et al. Linkage disequilibrium and association of MAPT H1 in Parkinson disease. Am J Hum Genet. 2004; 75(4):669-77. PMID: 15297935

15. Allen M, Kachadoorian M, Quicksall Z, Zou F, Chai HS, Younkin C, et al. Association of MAPT haplotypes with Alzheimer's disease risk and MAPT brain gene expression levels. Alzheimers Res Ther. 2014; 6(4):39. doi: 10.1186/alzrt268 PMID: 25324900

16. Ghidoni R, Signorini S, Barbiero L, Sina E, Cominelli P, Villa A, et al. The H2 MAPT haplotype is associated with familial frontotemporal dementia. Neurobiol Dis. 2006; 22(2):357-62. PMID: 16410051

17. Trabzuni D, Wray S, Vandrovcova J, Ramasamy A, Walker R, Smith $C$, et al. MAPT expression and splicing is differentially regulated by brain region: relation to genotype and implication for tauopathies. Hum Mol Genet. 2012; 21(18):4094-103. doi: 10.1093/hmg/dds238 PMID: 22723018

18. Bennett DA, Schneider JA, Arvanitakis Z, Wilson RS. Overview and findings from the religious orders study. Curr Alzheimer Res. 2012; 9(6):628-45. PMID: 22471860

19. Bennett DA, Schneider JA, Buchman AS, Barnes LL, Boyle PA, Wilson RS. Overview and findings from the rush Memory and Aging Project. Curr Alzheimer Res. 2012; 9(6):646-63. PMID: 22471867

20. Corneveaux JJ, Myers AJ, Allen AN, Pruzin JJ, Ramirez M, Engel A, et al. Association of CR1, CLU and PICALM with Alzheimer's disease in a cohort of clinically characterized and neuropathologically verified individuals. Hum Mol Genet. 2010; 19(16):3295-301. doi: 10.1093/hmg/ddq221 PMID: 20534741

21. Wider C, Vilarino-Guell C, Jasinska-Myga B, Heckman MG, Soto-Ortolaza AI, Cobb SA, et al. Association of the MAPT locus with Parkinson's disease. Eur J Neurol. 2010; 17(3):483-6. doi: 10.1111/j.14681331.2009.02847.x PMID: 19912324

22. Schroeder A, Mueller O, Stocker S, Salowsky R, Leiber M, Gassmann M, et al. The RIN: an RNA integrity number for assigning integrity values to RNA measurements. BMC Mol Biol. 2006; 7:3. PMID: 16448564

23. Trapnell C, Pachter L, Salzberg SL. TopHat: discovering splice junctions with RNA-Seq. Bioinformatics. 2009; 25(9):1105-11. doi: 10.1093/bioinformatics/btp120 PMID: 19289445

24. Braak H, Del Tredici K, Rub U, de Vos RA, Jansen Steur EN, Braak E. Staging of brain pathology related to sporadic Parkinson's disease. Neurobiol Aging. 2003; 24(2):197-211. PMID: 12498954

25. Schneider JA, Li JL, Li Y, Wilson RS, Kordower JH, Bennett DA. Substantia nigra tangles are related to gait impairment in older persons. Ann Neurol. 2006; 59(1):166-73. PMID: 16374822

26. Bennett DA, Wilson RS, Schneider JA, Evans DA, Aggarwal NT, Arnold SE, et al. Apolipoprotein E epsilon4 allele, AD pathology, and the clinical expression of Alzheimer's disease. Neurology. 2003; 60 (2):246-52. PMID: 12552039

27. Caffrey TM, Wade-Martins R. The role of MAPT sequence variation in mechanisms of disease susceptibility. Biochem Soc Trans. 2012; 40(4):687-92. doi: 10.1042/BST20120063 PMID: 22817717

28. Louis ED, Schupf N, Manly J, Marder K, Tang MX, Mayeux R. Association between mild parkinsonian signs and mild cognitive impairment in a community. Neurology. 2005; 64(7):1157-61. PMID: 15824340

29. Uemura Y, Wada-Isoe K, Nakashita S, Nakashima K. Depression and cognitive impairment in patients with mild parkinsonian signs. Acta Neurol Scand. 2013; 128(3):153-9. doi: 10.1111/ane.12089 PMID: 23397887 
30. Iwata A, Nagata K, Hatsuta H, Takuma H, Bundo M, Iwamoto K, et al. Altered CpG methylation in sporadic Alzheimer's disease is associated with APP and MAPT dysregulation. Hum Mol Genet. 2014; 23 (3):648-56. doi: 10.1093/hmg/ddt451 PMID: 24101602

31. Coupland KG, Mellick GD, Silburn PA, Mather K, Armstrong NJ, Sachdev PS, et al. DNA methylation of the MAPT gene in Parkinson's disease cohorts and modulation by vitamin E in vitro. Mov Disord. 2014; 29(13):1606-14. doi: 10.1002/mds.25784 PMID: 24375821

32. Hayesmoore JB, Bray NJ, Cross WC, Owen MJ, O'Donovan MC, Morris HR. The effect of age and the H1c MAPT haplotype on MAPT expression in human brain. Neurobiol Aging. 2009; 30(10):1652-6. doi: 10.1016/j.neurobiolaging.2007.12.017 PMID: 18276036

33. Caffrey TM, Joachim C, Wade-Martins R. Haplotype-specific expression of the N-terminal exons 2 and 3 at the human MAPT locus. Neurobiol Aging. 2008; 29(12):1923-9. PMID: 17602795

34. Karch CM, Jeng AT, Goate AM. Extracellular Tau levels are influenced by variability in Tau that is associated with tauopathies. J Biol Chem. 2012; 287(51):42751-62. Epub 2012/10/30. doi: 10.1074/jbc. M112.380642 PMID: 23105105; PubMed Central PMCID: PMCPMC3522274.

35. Myers AJ, Pittman AM, Zhao AS, Rohrer K, Kaleem M, Marlowe L, et al. The MAPT H1c risk haplotype is associated with increased expression of tau and especially of 4 repeat containing transcripts. Neurobiol Dis. 2007; 25(3):561-70. PMID: 17174556

36. Caffrey TM, Joachim C, Paracchini S, Esiri MM, Wade-Martins R. Haplotype-specific expression of exon 10 at the human MAPT locus. Hum Mol Genet. 2006; 15(24):3529-37. PMID: 17085483

37. Chambers CB, Lee JM, Troncoso JC, Reich S, Muma NA. Overexpression of four-repeat tau mRNA isoforms in progressive supranuclear palsy but not in Alzheimer's disease. Ann Neurol. 1999; 46 (3):325-32. PMID: 10482263 